Proceeding

\title{
Monitoring of patient status during radiotherapy treatment
}

Keywords: daily communication, patient monitoring, changes and side effects during the treatment plan, documented monitoring of the patient

\section{Objective and aims}

Daily communication with the patient during the repeated and prolonged treatment causes RT radiotherapy technicians and obligation to monitor the patient's condition, namely how patients tolerate radiotherapy treatment, whether there are changes and side effects, and in what form and to inform the radiation oncologist. In Serbia, there is no specific supporting document that radiotherapy technician used for this purpose. The aim is to present, explain, and in the near future is accepted as an integral part, List of monitoring the patient during radiotherapy treatment, led by radiotherapy technician.

\section{Methodology}

Daily communication carries with it a common question that radiotherapy technician puts the patient, How Are You? To answer the question more parameters relevant to the condition of the patient during the treatment: a subjective condition, condition assessment, whether the patient complains of certain ailments - fatigue, nausea, diarrhea, dry mouth, throat etc. All of these phenomena and changes in the patient's condition are possible side effects during radiotherapy treatment. All of these side effects are possible to prevent or keep under control in aim of successful implementation of radiotherapy treatment. That's one of main skills and responsibilities of job Radiotherapy Technicians .Everyday Radiotherapy technician checks the status of the patient's skin, whether there erythema. Everything this must be recorded in the "monitoring list".

Monitoring list must have enough rows and columns. That means to cover and include all radiation side effects who can appear during treatment. Monitoring list can be in standard paper form or electronic form, or best in booth form. All Radiotherapy centers using information system who owns specific folders for patient data, and that's very helpful for monitoring list. Also, couple producers of equipment offer specific systems for patient monitoring who designed the operating system platforms available on mobile devices. That's systems is very useful for direct communication between the patient
Volume 7 Issue 2 - 2017

Ilija Curic

Institute for Oncology and Radiology of Serbia, Serbia

Correspondence: Ilija Curic, President of Serbian Society of Radiotherapy Technicians, Institute for Oncology and Radiology of Serbia, Serbia, Email iccurici@gmail.com

Received: December 13, 2016 | Published: January 18, 2017

and Radiotherapy technicians. Using that system booth side -staff and patient- gain insight into the condition of the patient.

\section{Result}

Primary documented monitoring of the patient, which is important for treatment and with regular check-ups with a radiation oncologist in the interest of the patient's status. It also provides an insight on the effects of therapy and possible adjustments in planning and implementing therapy.

\section{Conclusion}

The monitoring of the patient during radiotherapy treatment in the interest of the patient and the radiotherapy team. Patient is paid necessary attention and treatment; a radiotherapy team documented correctly applies their skills and knowledge in the interest of the patient.

\section{Acknowledgments}

None.

\section{Conflicts of interest}

Author declares there are no conflicts of interest. 